\title{
The Evolution of Galaxies in Compact Groups
}

\author{
Roger Coziol ${ }^{1,2}$, Reinaldo R. de Carvalho ${ }^{2}$ \\ Hugo V.Capelato ${ }^{3}$ \\ and
}

André L. B. Ribeiro ${ }^{4}$

Received

accepted

\footnotetext{
${ }^{1}$ Laboratório Nacional de Astrofísica - LNA/CNPq, Rua Estados Unidos, 154, Bairros das Nações - 37500-000 - Itajubá, MG, Brasil

${ }^{2}$ Observatório Nacional, Rua Gal. José Cristino, 77 - 20921-400, Rio de Janeiro, RJ., Brasil

${ }^{3}$ Divisão de Astrofísica - INPE/MCT, C.P. 515 - 12201-970, S. José dos Campos, SP., Brasil

${ }^{4}$ Dept. Matemática Aplicada - IMECC, Universidade E. de Campinas, 13083-970 SP, Brasil
} 


\begin{abstract}
We present the analysis of the spectra of 62 galaxies in 15 compact groups. The galaxies were classified in four activity classes: galaxies without emission, starburst galaxies, luminous AGNs (Seyfert and LINERs) and low-luminosity AGNs (LLAGNs).

The star formation in the HCG starbursts is more intense than in normal spirals, but comparable to those observed in starburst nucleus galaxies (SBNGs) in the field. In general, the HCG starbursts have mean solar gas metallicity and do not follow the metallicity-luminosity relation traced by the early-type SBNGs in the field, suggesting that most of them are late-type SBNGs. This morphology preference coupled to the observation that the HCG starbursts are predominantly in the halo of the groups is consistent with the idea that compact groups are embedded in sparser structures.

The stellar metallicities of the non starburst galaxies are comparable to those observed in normal galaxies with similar morphologies, but are relatively high for their luminosities. In these galaxies the metal absorption lines equivalent widths are slightly narrower than normal while the Balmer absorption lines are relatively strong. All these observations suggest the presence of a population of intermediate--age stars. These galaxies could be "post-starburst", but at a very advanced stage of evolution. The last bursts happened more than 2 Gyrs in the past.
\end{abstract}

Our observations are supporting a scenario where the core of the groups are slowly collapsing evolved systems embedded in more extended structures (Ribeiro et al. 1998). In the core of the groups, the interactions were more frequent and the galaxies evolved at a more rapid rate than in their halos. 
Subject headings: galaxies: Compact groups - galaxies: Evolution - galaxies:

Interactions - galaxies: AGNs - galaxies: Starbursts 


\section{Introduction}

One of the most important problems in cosmology concerns the formation and evolution of galaxies. Recent works support the hierarchical galaxy formation theory, where massive galaxies form by subsequent mergers of smaller mass of gas and star systems (Larson 1990; Clements \& Couch 1996; Baugh et al. 1996; Rauch, Haehnelt \& Steinmetz 1997). Although the details are still largely missing, it seems that the starburst phenomenon could be an important phase of this process (Sofue \& Habe 1992; Kennicutt 1994; Clements \& Couch 1996; Cowie et al. 1996; Simard \& Pritchet 1997; Lowenthal et al. 1997; Coziol et al. 1997a). In the starburst-interaction scenario, tidal forces produced by interaction are sufficient to trigger global bursts of star formation resulting in changes of the stellar populations, chemical abundances and even morphologies of the galaxies (Toomre \& Toomre 1972; Tinsley \& Larson 1980; Kauffmann et al. 1996; Lavery et al. 1996; Neuschaefer, Ratnatunga \& Griffiths 1997; Coziol 1997). By considering various mechanisms for transferring matter down to the nucleus of the galaxies, one could extend the starburst-interaction scenario to include the formation and evolution of AGNs (Weedman 1983; Sanders et al. 1988; Carlberg 1990; Omont et al. 1996; Bahcall et al. 1997).

A natural consequence of the starburst-interaction scenario is that the evolution of galaxies should depend on the environment in a systematic way, being more effective in a dense, low-velocity dispersion region. One of the most important piece of evidence supporting the environment dependence is the segregation of morphologies observed in galaxy clusters (Dressler 1980). It is not clear however, how and when this distinction occurs. Although cluster ellipticals seem to be old and quietly evolving galaxies (Schade, Barrientos \& Lopez-Cruz 1997; Ellis et al. 1997; Bender et al. 1997), the history of the cluster spiral galaxies could be much different, experiencing more evolution until a recent epoch (Butcher \& Oemler 1978; Larson, Tinsley \& Caldwell 1980; Caldwell \& Rose 1997; 
Stein 1997; Abraham et al. 1997; Dressler \& Smail 1997).

In this context of the formation and evolution of galaxies, Hickson's Compact Groups (HCGs) of galaxies (Hickson 1982) are somehow puzzling. These groups combine high spatial densities and small velocity dispersions suggesting rapid merging rates and possibly enhanced star formation and nuclear activity (Hickson 1990; Rubin et al. 1991). Yet, the merging rates seem to be lower than that expected from the observed crossing times (Zepf \& Whitmore 1991; Zepf 1993) and the star formation and AGN activity are at a relatively low level (Coziol et al. 1997b, hereafter Paper 1; Leon et al. 1998; Verdes-Montenegro et al. 1998). As an alternative, it was suggested that the HCGs are the result of chance alignments of galaxies in larger structures (Mamon 1994; Ostriker et al. 1995). But, this hypothesis seems to be ruled out based on the most recent X-ray survey which suggests that as much as $75 \%$ of these systems present extended emission from hot intragroup gas (Ponmam et al. 1996).

A more satisfactory answer to the ambiguities presented by the HCGs comes from a new spectroscopic survey of galaxies performed by de Carvalho et al. (1997). The dynamical analysis based on this survey (Ribeiro et al. 1998; Zepf, de Carvalho \& Ribeiro 1997) reveals that most of the groups are part of larger structures and represent different dynamical stages of evolution. Following the discovery of a significant number of low-luminosity AGNs (LLAGNs) in HCGs, we verified that the core of the groups are dominated by AGNs and galaxies without emission, whereas the starbursts galaxies are mostly at the periphery (Paper 1). From this result, we propose a scenario where the type of activity (AGN or starburst) in galaxies correlates to a morphology-density evolution. The present contribution presents other evidences in favor of this scenario and in support of the idea of a replenishment of the groups by spiral galaxies during their formation. If CGs are physically bound systems evolving on a timescale of $0.1 \mathrm{H}_{\circ}^{-1}$, then the first generation of these 
groups was already converted into a field elliptical galaxy. Therefore, some replenishment mechanism must operate in order to keep the number of groups approximately constant (Diafero, Geller \& Ramella 1994, 1995; Governato, Tozzy \& Cavaliere 1996; Ribeiro et al. 1998).

\section{Results of the spectral analysis}

In order to study the nature of compact groups of galaxies, de Carvalho et al. (1997) obtained the spectra of 316 galaxies in the regions of a selected sample of 17 HCGs, using the ARGUS fiber-fed spectrograph at the 4m CTIO telescope. The details on the instrumental setup and a discussion of the data reduction can be found in de Carvalho et al. (1997). From this sample, we selected 82 galaxies which have a spectrum with a signal to noise ratio sufficiently high to study their spectral characteristics. Of these galaxies, 28 (34\%) present only absorption lines and 54 (66\%) present both emission and absorption lines. A first paper (Coziol et al. 1997b; hereafter Paper I) presented a classification of the activity types of the emission-line galaxies. In the present contribution, we extend our analysis by studying the absorption-line features of the galaxies which are group members.

In Paper I, we have distinguished between the galaxies which are real members of the HCGs from those which are on the foreground or background. We note that many of these "non-group members" could reside in other bound structures (Ribeiro et al. 1998). Because our observations on these possible structures are very incomplete, we choose not to include them in the present study. Our final sample is composed of 62 galaxies which are members of 15 compact groups.

In Table 1, we present the line intensities of the HCG emission-line galaxies. The first column gives the identification of the galaxies following de Carvalho et al. (1997). 
In columns 2 to 12 , the intensities are given relatively to $\mathrm{H} \alpha=100$. The uncertainties were determined from Poisson statistics. Errors smaller than $1 \%$ are not indicated. The lines were measured by fitting a gaussian profile after the subtraction of a template galaxy spectrum. This technique was applied systematically to correct for the presence of strong Balmer absorption lines observed in a significant fraction of the galaxies in our sample. Details on how this subtraction was performed can be found in Paper I.

In Table 2, we summarize the results of our classification of the activity type of the HCG emission-line galaxies as determined in Paper I and list some of their basic characteristics. The B magnitudes in column 2 and the redshifts in column 3 were taken from de Carvalho et al. (1997). In column 4, we list the absolute magnitudes of the galaxies, assuming $\mathrm{H}_{0}=75 \mathrm{~km} \mathrm{~s}^{-1} \mathrm{Mpc}^{-1}$. The morphological types listed in column 5 were taken from Mendes de Oliveira \& Hickson (1994). The different types of activity in column 6 are described as: starburst galaxies [SBNGs or HII], AGNs (Seyfert 2 [Sy2] or LINER [LNR]) and low-luminosity AGNs [dSy2 or dLNR]. Column 7 gives the $\mathrm{H} \alpha+[\mathrm{N}$ II] equivalent width $(\mathrm{EW})$.

The absorption features identified in the spectra of the galaxies were measured by drawing the pseudo continuum at the highest points, using a region $\sim 100 \AA$ wide on each side of the line (Zabludoff et al. 1996). This method was used because the spectra are not flux calibrated and most of our galaxies are early-type galaxies with a spectrum full of faint absorption lines. For the Balmer lines, we did not use a narrow continuum window definition like in the Lick system (Faber et al. 1989) because this method clearly underestimate the equivalent width of broad lines which are numerous in our spectra (Worthey et al. 1994). We expect our method to yield slightly overestimated EWs and peak intensities as compared to values obtained using the Lick system.

The determination of the Mg2 index follows the definition of Burstein et al. (1984), 
using their windows for the identification of the pseudo continuum. In Jorgensen (1997) we have found 6 HCG galaxies in common with our sample, where the Mg2 index was measured in the Lick system. The values reported by Jorgensen show an offset of -0.11 as compared to ours. This difference probably reflects the fact that Jorgensen's measurements were done with better resolution. Thus, we have taken the offset of -0.11 to correct our Mg2 measurements.

In Tables 3 to 6 , we present the absorption line characteristics of the galaxies exhibiting different activity types. Columns 2 to 9 give the EW of the most prominent absorption features. The mean uncertainty of $\pm 0.5 \AA$ was determined by comparing values obtained in two different spectra of the same object. Column 10 gives the ratio of the center of the line intensity of the $\mathrm{Ca}$ II $\mathrm{H}+\mathrm{H} \epsilon$ lines to the center of the line intensity of the Ca II $\mathrm{K}$ and column 11 gives the $\mathrm{Mg}_{2}$ index. Table 6 has an extra column which gives the morphological type of the non-emission galaxies.

\section{The nature of the starburst in the HCGs}

In Figure 1, we compare the mean FWHM of the emission-lines identified in the spectra of the galaxies with their $\mathrm{H} \alpha+[\mathrm{N}$ II] EWs. On average, the AGNs (that is, the LLAGNs and the luminous AGNs) have smaller EWs and relatively broader FWHM than the starburst galaxies. The AGNs in our sample present FWHM which are within the range of typical observed values (Osterbrock \& Mathews 1986). Some of the starburst galaxies have unusually broad FWHM for H II regions, but comparable to those observed in Luminous Infrared Galaxies (Veilleux et al. 1995).

Because our spectra are not flux calibrated we cannot determine directly the star formation rates in the HCG starbursts. However, we can get a qualitative estimate of the 
level of star formation activity in these galaxies by examining their $\mathrm{H} \alpha+[\mathrm{N}$ II] EWs. It is well known, in particular, that galaxies with very high equivalent width $(\geq 50 \AA)$ invariably prove to be galaxies undergoing intense star formation (Kennicutt \& Kent 1983). In Figure 2, we compare the distribution of the $\mathrm{H} \alpha+[\mathrm{N} \mathrm{II}]$ EWs of the HCG starbursts to those observed in normal spirals (Kennicutt 1983). The HCG starbursts usually have relatively high EWs values, suggesting that the star formation in these galaxies is on average more intense than in normal spiral galaxies. This observation is important because it means that we cannot relate the star formation activity in these galaxies to normal star formation occurring in the disk of late-type galaxies. This alternative was recently proposed by Rakos, Maindl \& Schombert (1996) to explain the Butcher-Oemler effect in rich clusters of galaxies at higher redshifts. In the HCGs starbursts, the star formation rates seem higher than normal, although comparable to those observed in starburst galaxies in the field.

Table 7 gives the gas densities and metallicities of the starburst galaxies in our sample. Column 2 shows the ratio $[\mathrm{S}$ II] $\lambda 6717 /[\mathrm{S}$ II] $\lambda 6731$ from which we deduce the electron densities, in column 3, assuming a gas temperature of $10^{4} \mathrm{~K}$ (Osterbrock 1989). The densities are comparable to those found in giant H II regions in the nucleus of galaxies. The metallicities, in column 4, were determined based on the metallicity-calibrated diagnostic diagram established by Coziol et al. (1994) and presented in Figure 3. The mean uncertainty on the metallicities is 0.2 dex. In Figure 3, the metallicities of the HCG starbursts are compared to those of a sample of Starburst Nucleus Galaxies (SBNGs) observed in the field (Coziol et al. 1997a, 1997c). As it can be seen, the mean metallicities of the HCG starbursts are comparable to those of the SBNGs in the field.

In Coziol et al. (1997a) it was shown that in the field the late-type SBNGs are 0.2 dex more metal rich on average than the early-type SBNGs. This difference in metallicity was explained by assuming different gas accretion rates during the formation of the galaxies: 
the late-type SBNGs have accreted more gas than stars than the early-type SBNGs (Coziol et al. 1997a). In Paper I, we noted that most of the HCG starbursts in our sample with a well-defined morphology are late-type galaxies. In Figure 3, we see that the mean metallicity of the HCG starbursts is close to solar. The HCG starbursts look, consequently, more similar to the late than to the early-type SBNGs. This is another argument suggesting that most of the HCG starbursts in our sample are in late-type spiral galaxies. To test this hypothesis, we have estimated the difference $\left([\mathrm{O} / \mathrm{H}]_{t h}-[\mathrm{O} / \mathrm{H}]_{o b s}\right)$ between the metallicity predicted by the luminosity-metallicity relation followed by the early-type SBNGs (Coziol et al. 1997a) and the observed metallicity of the HCG starbursts. In Figure 4, we can see that the metallicities of the HCG starbursts generally diverge from the values predicted by the luminosity-metallicity relation. The distribution of the differences is similar to the one observed for the late-type SBNGs in the field. A Kolmogorov-Smirnov test indicates that there is no difference between the Late-type SBNGs and HCG starburst distributions at the $5 \%$ significance level. Therefore, unless they are completely different from those in the field, most of the HCG starbursts in our sample must be late-type SBNGs.

\section{The nature of the stellar population in the HCG galaxies}

The study of the absorption lines in galaxies allows to determine the nature of the dominant stellar populations. By comparing the stellar populations in the HCG galaxies with those found in normal galaxies in the field we can verify if the evolution of these galaxies was influenced by their environment. But before doing such a comparison, we have to establish what is the observed pattern for normal galaxies, starburst and AGN galaxies in the field.

For normal galaxy spectra, the absorption features vary with their morphologies. This is due to a variation of the nature of the dominant stellar population in galaxies with 
different morphologies (Hamilton 1985; Rose 1985; Kennicutt 1992; Zaritsky, Zabludoff \& Willick 1996). In general, the EWs of different absorption lines trace a continuous sequence which correlates to morphology. This phenomenon is illustrated in Figure 5a where we show the behavior for the EW of the Ca II K line as a function of the EW of the G-band in a sample of normal spiral galaxies observed by Kennicutt (1992). The EWs were measured here by Jablonka \& Alloin (1995). The EWs increase from the late-type to the early-type galaxies and follow the increases of age of the dominant stellar population.

In normal spiral galaxies, the dominant stellar population changes from the disk to the bulge. The stellar populations are generally older in bulges than in disks. This is illustrated in Figure 5b, where we show the behavior of the EW of the Ca II K line and of the G-band lines, as measured in the bulges of spiral galaxies by Jablonka et al. (1996). The EW of these two lines are comparable to those observed in elliptical galaxies (Jablonka et al. 1996). This result is important as it suggests that the bulk of the stars in the bulges probably formed by similar processes than in elliptical galaxies (Jablonka et al. 1996).

In the starburst and AGN galaxies, the change in the absorption line features with the morphologies are different than in the normal spiral galaxies. In Figure 5b, we show the relation of the EW of the Ca II K line with the one for the G-band, as measured in a sample of starburst galaxies by Storchi-Bergmann, Calzetti \& Kinney (1994). The low values of the EWs suggest that the spectra of the starbursts are dominated by young stellar populations, regardless of their morphologies. Therefore, we cannot determine the morphology of a starburst galaxy by the absorption features in its spectrum.

For the AGNs, the situation is more ambiguous than for the starbursts. In Figure 5c, we show the EW of the Ca II K line and of the G-band, as measured in a sample of Seyfert galaxies by Storchi-Bergmann, Kinney \& Challis (1995). In general, the Seyfert galaxies seem to follow the same morphological sequence as normal galaxies, but the dispersion 
in each morphological bin is large. In this kind of analysis we should consider a possible dilution effect due to the underlying featureless continuum of the AGN. Recently Cid Fernandes, Storchi-Bergman \& Schmitt (1998) have shown that in the optical a dilution effect is observed only in Seyfert 1, not in Seyfert 2. In this latter case, therefore, the high dispersion of EWs values observed probably reflects the fact Seyferts 2 do not constitute, from the point of view of star formation, a homogeneous class of galaxies: some of these galaxies could be starburst while others are normal. This interpretation is supported by the recent observations showing intrinsic differences between the two types of Seyferts (see González Delgado et al. (1997) and Malkan, Gorjian \& Tam (1998)). The consequence for our analysis is that when an AGN is present the EWs of the galaxies are not clearly related to their morphologies.

From the previous discussion, we discriminate in Figure 5d three different regions related to three dominant stellar populations in galaxies: early-type galaxies, [E], which are dominated by an old stellar population; intermediate and late-type spirals, [L], which are dominated by intermediate-age and young stellar populations; and starburst galaxies, [SB], which are dominated by young and massive stars, independent of their morphologies. Having determined these regions, we can proceed to compare the stellar population in our sample of HCG galaxies with those of normal galaxies in the field. Figure 5d shows the results for the HCG galaxies with different activity types. The behaviors of the HCG starbursts and AGNs are consistent with the general trend observed for galaxies of their type. On the other hand, the HCG galaxies without emission and the LLAGNs all have smaller EWs than normal galaxies for their morphologies. This is surprising, because most of these galaxies are early-type and, consequently, we would have expected to find them clearly located in the E box.

In order to verify that this phenomenon is not due to spurious measurements of some 
absorption lines (the Ca II K line, for instance, is very near the border of our spectra and consequently hard to measure), we show in Figure 6 the same kind of analysis using the CN band. The behavior is the same: the non-emission galaxies and the LLAGNs have smaller EWs than normal galaxies for their morphologies. Note that following our methodology we expect to overestimate the EW values, which means that the real effect could even be more pronounced than what we have measured.

In Figures 5 and 6, there seem to be no difference between the stellar populations of the non-emission line galaxies and the LLAGNs. It suggests that the AGN continuum in the LLAGNs has no effect on the EWs absorption lines due to their stellar population (the continuum of the AGN is probably too weak as already pointed out in Paper I). It seems, therefore, that the only way to explain the small EW values in all these galaxies is to assume the presence of younger stellar populations than usually observed in galaxies with similar morphologies.

To test this hypothesis, we examine in Figure 7 the behavior of the EW of $\mathrm{H} \delta$ as a function of the ratio ( $\mathrm{Ca}$ II $\mathrm{H}+\mathrm{H} \epsilon$ )/Ca II $\mathrm{K}$. The increase of the EW of the Balmer lines due to the presence of young stellar populations is already well established and documented (Dressler \& Shectman 1994; Leonardi \& Rose 1996; Caldwell et al. 1996; Zabludoff et al. 1996; Poggianti \& Barbaro 1996; Barbaro \& Poggianti 1997). The behavior of the (Ca II $\mathrm{H}+\mathrm{H} \epsilon) / \mathrm{Ca}$ II $\mathrm{K}$ ratio due to the evolution of stellar populations is explained by Rose (1985). This parameter was used recently by Leonardi \& Rose (1996) to estimate the age of post-starburst galaxies in Coma. In Figure 7, we can see that the majority of the non-emission and LLAGN galaxies in our sample have higher values of EWs than normal galaxies for their morphologies. A mean value of $\operatorname{EW}(\mathrm{H} \delta)=1.7$ is considered normal for late type spirals while $\mathrm{EW}(\mathrm{H} \delta)=2.5$ is considered unusually "strong" (Poggianti \& Barbaro 1996, 1997; Zabludoff et al. 1996). 
Note that the EW(H $\delta)$ values found for HCG post-starburst galaxies seem relatively low in comparison with those measured in post-starburst galaxies found in the literature. This suggests that the "post-starburst" phase in the HCG galaxies is at a very advanced stage of evolution. This interpretation is consistent with the observed ratios (Ca II H + $\mathrm{H} \epsilon$ )/Ca II K, which indicate a fraction of A type stars lower than 30\% (Rose 1985). Using the burst models of Poggianti \& Barbaro (1996) and Barbaro \& Poggianti 1997, a rough estimate would place the last star formation burst more than 2 Gyrs in the past.

\section{Stellar metallicities of the galaxies in the compact groups}

In this section, we compare the stellar metallicities of HCG galaxies with those observed in normal galaxies (that is, non starburst and non AGN galaxies) with different morphologies. Our analysis is based on the $\mathrm{Mg}_{2}$ index which is generally used to determine the metallicity of the stellar population in composite systems (Burstein et al. 1984; Brodie \& Huchra 1990; Worthey, Faber \& González 1992).

In Figure 8a, we show the distribution of the $\mathrm{Mg}_{2}$ index as a function of the morphological type in normal galaxies. The normal galaxies population is represented by elliptical and lenticular galaxies observed by Bender et al. (1993) and by a sample of spiral and lenticular bulges of galaxies observed by Jablonka et al. (1996). As expected, the normal galaxies trace a sequence of increasing metallicities, going from the late-type spirals to the early-type galaxies. A similar sequence is observed for the metallicity of the gas in the spiral galaxies (Zaritsky et al. 1996).

The $\mathrm{Mg}_{2}$ index for the HCG galaxies are presented in Figure 8b. In general, the stellar metallicities in the HCG starbursts are comparable to those observed in the bulge of the late-type spiral galaxies. This reinforces our thesis that the HCG starbursts are mostly 
late-type spirals. The stellar metallicities of all the other non starburst HCG galaxies are also comparable to those observed in galaxies with similar morphologies. Note however that the average metallicity is high. In particular, the late-type HCG galaxies seem to have metallicities comparable to those of the early-type. The tendency of high metallicities for the late-type HCG galaxies in the HCG galaxies is better seen in Figure 9, where we present the $\mathrm{Mg}_{2}$ indices as a function of the absolute magnitudes of the galaxies. On average, the $\mathrm{Mg}_{2}$ index is $0.04 \mathrm{mag}$ higher for the HCG non starburst galaxies than for early-type galaxies with similar luminosities. In Figure 9, we have also reported the values of $\mathrm{Mg}_{2}$ as measured by Jorgensen (1997) which show the same effect. Using the relation [Fe/H] $=9.92 \times \mathrm{Mg}_{2}-2.21$, as proposed by Brodie \& Huchra (1990), we found an excess of 0.4 dex in metallicities.

\section{Discussion}

The main results of our analysis of the emission and absorption features of the galaxies in the compact groups may be summarized as follows:

1. Most of the HCG starbursts are late-type spirals. The star formation in these galaxies is higher than in normal spirals, but comparable to those found in starburst galaxies in the field. Compared to the other types of galaxies in the groups, the HCG starbursts have slightly lower metallicities, suggesting that their evolution was somehow different compared to the other galaxies in the group.

2. The non-emission line galaxies and the LLAGNs show unusually small equivalent widths and strong balmer lines for their morphologies. This suggests the presence of intermediate-age stellar population. However, the fraction of these stars seem to be much lower than in post-starburst galaxies found in the literature, suggesting a more 
advanced stage of evolution.

3. All the non starburst HCG galaxies have $\mathrm{Mg}_{2}$ indices which are higher than for normal galaxies for their luminosities. In terms of metallicity $([\mathrm{Fe} / \mathrm{H}])$, this would correspond to an excess of almost 0.4 dex.

Our observations are consistent with a scenario where the formation and evolution of galaxies in the compact groups are regulated by their environment. Merger, starbursts and/or gas stripping are probably the main processes behind the formation and evolution of these systems. Evidence of gas stripping is inferred from the fact that HCG galaxies are generally deficient in HI (Huchtmeier 1997; OOsterloo \& Iovino 1997). This process seems also a reasonable mechanism to explain the presence of diffuse X-ray emission observed in the groups (Ponman et al. 1996). The gas stripping mechanism would also produce false post-starburst galaxies by truncation of star formation: the constant star formation of a spiral is artificially stopped due to the rapid depletion of the gas favoring the detection of the intermediate age stellar population. Skillman et al. (1996) have also suggested that the curtailment of the metal poor gas infall produced by gas stripping would contribute to increase substantially the gas metallicity of these galaxies. Note however, that in the HCG galaxies it is the stars which seem to be more metal rich. This implies that these stars were formed from previous star formation events, or that these galaxies are real post-starburst (similar phenomena is observed in Coma, see Caldwell \& Rose 1997). This is consistent with the observations by Verdes-Montenegro et al. (1997) which show CO depletion in the HCG galaxies. As noted by these authors, gas exhaustion by tidally induced star formation is probably the only way to produce the depletion of molecular gas, since tidal stripping is less likely for molecular gas concentrated in the inner disks. Other elements in favor of past induced star formation either by merger or interactions are the observations, also by Verdes-Montenegro et al. (1997), of the presence of molecular gas in early-type galaxies 
(like in HCG 90 and HCG 86) and also the fact that the galaxies deficient in molecular gas are located in the most compact, and consequently dynamically most evolved groups.

But the strongest argument in favor of the role of merger for galaxy evolution in groups is the density-morphology-activity trend observed in HCGs (see Paper I): the most massive and early-type galaxies are AGNs or non-emission galaxies, mainly located in the core of the groups. In Ribeiro et al. (1998), it was noted that most of the HCGs are embedded in larger structures forming extended halos around more dense and dynamically distinct cores displaying low velocity dispersions. Figure 10 shows the frequency distribution of peculiar velocities of the HCG galaxies of our sample relative to their group mean velocity. There is a clear tendency for the AGNs and galaxies without emission to have smaller peculiar velocities than the starbursts. Now, not only these galaxies have early-type morphologies but they are also more metal rich than the starburst galaxies which are predominantly in the halos. Therefore, the the cores are in a more advanced stage of evolution than the halos. In the cores, because of their high density and low peculiar velocities, the interactions are more frequent and the galaxies evolve more rapidly than in the halos of the groups.

The fact that the galaxies in the core of the groups are more evolved than those in the halo only exacerbate the low crossing time paradox related to the evolution of these structures. Indeed, our observations suggest that the last burst of star formation occurred more than 2 Gyr in the past and that the present rates of star formation in these galaxies are relatively low. These observations are inconsistent with the short merging and dynamical time scales. Numerical simulations indicate that these systems are dinamically unstable, with an orbital decay timescale between 0.01 and $0.1 \mathrm{H}_{\circ}^{-1}$ (Barnes 1985, 1989). One solution, other than assuming the presence of a large amount of dark matter, would be to suppose that the core of the groups formed relatively recently from the merging of smaller mass galaxies. One example of this process still going on today would be HCG 16 
(Ribeiro et al. 1996, Coziol \& de Carvalho 1998, in preparation).

In the halos of the groups, the interactions are less frequent due to the lower densities and the higher peculiar velocities of the galaxies, allowing the formation and survival of the spirals over longer time scales. In this sense the fact that all HCG starbursts may be late-type spirals could be very significant.

Our observations are consistent with a scenario where the core of the groups are slowly collapsing and evolved structures embedded in a more extended system (Ribeiro et al. 1998). The fact that spirals in the halo are starbursts suggests that there is still a lot of activity going on in the halo. Following this activity, some of the starburst galaxies in the halo may fall into the core and enrich it in spirals.

We would like to thank the referee Dr. Gregory Bothun for his remarks and suggestions which greatly contributed to improve the quality of this paper. We also thank Dr. Eduardo Telles for stimulating discussions on the nature of the starburst galaxies. R. Coziol acknowledges the financial support of the CNPq, under contracts 650018/97-4 and A. L. B. Ribeiro acknowledges the support of the Brazilian CAPES. 


\section{REFERENCES}

Abraham, R. G., Smecker-Hane, T. A., Hutchings, J. B., Carlberg, R. G., Yee, H. K. C., Ellingson, E., Morris, S., Oke, J. B., Rogler, M. 1997, ApJ, 476, 7

Bahcall, J. N., Kirhakos, S., Saxe, D. H., Schneider, D. P. 1997, ApJ, 479, 642

Barbaro, G., Poggianti, B. M., 1997, A\&A, 324, 490

Baugh, C. M., Cole, S., Frenk, C. S., 1996, MNRAS, 282, 27

Bender, R., Burstein, Faber, S. M. 1993, ApJ, 411, 137

Bender, R., Saglia, R. P., Ziegler, B. 1997, in The Early Universe with the VLT, eds. J. Bergeron et al., Springler, in press

Brodie, J. P., Huchra, J. P. 1990, ApJ, 362, 503

Butcher, H., Oemler, A. 1978, ApJ, 219, 18

Caldwell, N., Rose, J. A., Franx, M., Leonardi, A. J. 1996, AJ, 111, 78

Caldwell, N., Rose, J. 1997, AJ, 113, 492

Carlberg, R. G. 1990, ApJ, 350, 505

Cid Fernandes, R., Storchi Bergmann, T. S., Schmitt, H. R. 1998, MNRAS, in press (astro-ph/9801309)

Clements, D. L., Couch, W. J. 1996, MNRAS, 280, 43

Cowie, L. L., Songaila, A., Hu, E. M., Cohen, J. G. 1996, AJ, 112, 839

Coziol, R. 1996, A\&A, 309, 345

Coziol, R. 1997, in Young Galaxies and QSO Absorption-line Systems, eds. S. M. Viegas, R. Gruewald, R. R. R. de Carvalho, PASP conf. series, Vol 114, p. 63

Coziol, R., Demers, S., Peña, M., Barneoud, R. 1994, AJ, 108, 405 
Coziol, R., Contini, T., Davoust, E., Considère, S. 1997a, ApJ, 481, L67

Coziol, R., Ribeiro, A. L. B., de Carvalho, R. R., Capelato, H. V. 1997b, ApJ, 493, 563, Paper I

Coziol, R., Demers, S., Barneoud, R., Peña, M. 1997c, AJ, 113, 1548

de Carvalho, R. R., Ribeiro, A. L. B., Capelato, H. V., Zepf, S. E. 1997, ApJS, 110, 1

Diafero, A., Geller, M. J., Ramella, M. 1994, AJ, 107, 868

Diafero, A., Geller, M. J., Ramella, M. 1995, AJ, 109, 2293

Dressler, A. 1980, ApJ, 236, 351

Dressler, A., Smail, I. 1997, in HST and the High Redshift Universe, eds. N. R. Tanvir, A. Aragon-Salamanca, J. V. Wall, World Scientific Press, in press

Ellis, R. S., Smail, I., Dressler, A., Couch, W. J., Oemler, Jr. A., Butcher, H., Sharples, R. M. 1997, ApJ, 483, 582

Faber, S. M., Wegner, G., Burstein, D., Davies, R. L., Dressler, A., Lynden-Bell, D., Terlevich, R. J. 1989, ApJ, 71, 173

González Delgado, R. M., Pérez, E., Tadhunter, C., Vilchez, J. M., Rodríguez-Espinoza, J. M. 1997, ApJS, 108, 155

Governato, F., Tozzi, P., Cavalieri, A. 1996; ApJ, 458, 18

Hamilton, D., 1985, ApJ, 297, 371

Hickson, P. 1982, ApJ, 255, 382

Huchtmeier, W. K., 1997, A\&A, 325, 473

Jablonka, P., Alloin, D. 1995, A\&A, 298, 361

Jablonka, P., Martin, P., Arimoto, N. 1996, AJ, 112, 1415

Jorgensen, I. 1997, MNRAS, 288, 161 
Kauffmann, G., Charlot, S., White, S. D. M. 1996, MNRAS, 283, 117

Kennicutt, Jr. R. C., Kent, S. M. 1983, AJ, 88, 1094

Kennicutt, Jr. R. C. 1992, ApJS, 79, 255

Kennicutt, Jr. R. C. 1994, in The Evolution of Galaxies and their Environment, eds. J. M. Shull, H. A. Thronson, Kluwer

Larson, R. B. 1990, PASP, 102, 709

Larson, R. B., Tinsley, B. M., Caldwell, C. N. 1980, ApJ, 237, 692

Lavery, R. J., Seitzer, P., Suntzeff, N. B., Walker, A. R., Da Costa, G. S. 1996, ApJ, 467, L1

Leon, S., Combes, F., Menon, T. K. 1998, A\&A, 330, 37

Leonardi, A. J., Rose, J. A. 1996, AJ, 111, 182

Lowenthal, J. D., Koo, D. C., Guzman, R., Gallego, J., Phillips, A. C., Faber, S. M., Vogt, N. P., Illingworth, G. D., Gronwall, C. 1997, ApJ, 481, 673

Malkan, M. A., Gorjian, V., Tam, R. 1998, ApJS, 117, in press (astro-ph/9803123)

Mamon, G. A. 1994, in Clusters of Galaxies, eds. F. Durret, A. Mazure, Tran Than Van, J., éditions frontières, p. 297

Mendes de Oliveira, C., Hickson, P. 1994, ApJ, 427, 684

Neuschaefer, L. W., IM, M., Ratnatunga, K. U., Griffiths, R. E., Casertano, S. 1997, ApJ, 480,59

Omont, A., Petitjean, P., Guilloteau, S., McMahon, S., Solomon, R. G., Pecontal, E. 1996, Nature, 382,428

OOsterloo, T., Iovino, A. 1997, PASA, 14, 48

Osterbrock, D. E. 1989, in Astrophysics of Gaseous Nebulae and Active Galactic Nuclei, University Science Book, Mill Valley, California 
Osterbrock, D. E., Mathews, G. 1986, ARA\&A, 24, 171

Ostriker, J. P., Lubin, L. M., Hernquist, L. 1995, ApJ, 444, L61

Poggianti, B. M., Barbaro, G. 1996, A\&A, 314, 379

Ponman, T. J., Borner, P. D. J., Ebeling, H., Böringer, H., 1996, MNRAS, 283, 690

Rakos, K. D., Maindl, T. I., Schombert, J. M. 1996, ApJ, 466, 122

Rauch, M., Haehnelt, M. G., Steinmetz, M. 1997, ApJ, 481, 601

Ribeiro, A. L. B., de Carvalho, R. R., Capelato, H. V., Zepf, S. E. 1998, ApJ, 497, 72

Rose, J. A. 1985, AJ, 90, 1927

Rubin, V. C., Hunter, D. A., Kent Ford, Jr. W. 1991, ApJS, 76, 153

Sanders, D. B., Soifer, B. T., Elias, J. H., Madore, B. F., Matthews, K., Neugebaueur, G., Scoville, N. Z. 1988, ApJ, 325, 74

Schade, D., Barrientos, L. F., Lopez-Cruz, O. 1997, ApJ, 477, 17

Simard, L., Pritchet, C. J. 1996, ApJ, in press (astro-ph/9606006)

Skillman, E. D., Kennicutt, Jr. R. C., Shields, G. A., Zaritsky, D. 1996, ApJ, 462, 147

Stein, P. 1997, A\&A, 480, 395

Sofue, Y., Habe, A. 1992, PASJ, 44, 325

Storchi-Bergmann, T., Calzetti, D., Kinney, A. L. 1994, ApJS, 429, 572

Storchi-Bergmann, T., Kinney, A. L., Challis, P. 1995, ApJS, 98, 103

Tinsley, B. M., Larson, R. B. 1979, MNRAS, 186, 503

Toomre, A., Toomre, J. 1972, ApJ, 178, 623

Veilleux, S., Kim, D. -C., Sanders, D. B., Mazzarella, J. M., Soifer, B. T. 1995, ApJS, 98, 171 
Verdes-Montenegro, L., Yun, M. S., Perea, J., del Olmo, A., Ho, P. T. P. 1998, ApJ, in press (astro-ph/9709121)

Weedman, D. W. 1983, ApJ, 266, 479

Worthey, G., Faber, S. M., González, J. J. 1992, ApJ, 398, 69

Worthey, G., Faber, S. M., Gonzalez, J. J., Burstein, D. 1994, ApJS, 94, 687

Zabludoff, A. I., Zaritsky, D., Lin, H., Tucker, D., Hashimoto, Y., Shectman, S. A., Oemler, A., Kirshner, R. P. 1996, ApJ, 466, 104

Zaritsky, D., Zabludoff, A. I., Willick, J. A. 1996, AJ, 110, 160

Zepf, S. E., Whitmore, B. C. 1991, ApJ, 383, 542

Zepf, S. E. 1993, ApJ, 407, 448

Zepf, S. E., de Carvalho, R. R., Ribeiro, A. L. B. 1997, ApJ, 488, L11 
Fig. 1.- Mean FWHM for the emission-line galaxies as a function of the $\mathrm{EW}(\mathrm{H} \alpha+[\mathrm{NII}])$. The domain of values for the FWHM usually observed in the narrow line regions of AGNs is indicated. The FWHM for the starbursts are unusually broad, but comparable to those observed in the Luminous Infrared Galaxies. In starbursts, the $\mathrm{EW}(\mathrm{H} \alpha+[\mathrm{NII}])$ is directly related to the star formation rates, which increase in the sense indicated by the arrow. Galaxies with $\log (\mathrm{EW}(\mathrm{H} \alpha+[\mathrm{NII}])>1.7$ are undergoing intense star formation (Kennicutt \& Kent 1983).

Fig. 2.- Frequency distribution of the $\mathrm{EW}(\mathrm{H} \alpha+[\mathrm{NII}])$ of the HCG starbursts. The HCG starburst are compared to the early and late-type spirals from the sample of Kennicutt \& Kent (1983). The mean and dispersion values are, $84 \pm 71$ for the HCG starbursts, $6 \pm 7$ for the early-type spirals and $33 \pm 20$ for the late-type spirals. $85 \%$ of the HCG starbursts have EWs higher than 20 and $70 \%$ have EWs higher than 40.

Fig. 3. - The diagnostic diagram of line ratios calibrated in metallicity (Coziol et al. 1994). The numbers correspond to different values of metallicities, as given by $12+\log (\mathrm{O} / \mathrm{H})$ (solar metallicity is 8.9 in this scale). The mean metallicities and dispersion for early-type and late-type SBNGs in the field are reported for comparison. The mean metallicity of the HCG starbursts is similar to those of the late-type SBNGs in the field.

Fig. 4.- The difference between the metallicities observed and the values predicted by the luminosity-metallicity relation followed by the early-type SBNGs in the field. In general, the HCG starbursts have metallicities that diverge from the luminosity-metallicity relation. The distribution of the differences is similar to the one for the late-type SBNGs in the field.

Fig. 5.- EW-EW diagram of the G-band as a function of the CaII K lines: a) Normal spirals show a sequence of increasing EW towards the early-type morphologies (Jablonka \& Alloin 1995); b) The bulge of spirals are dominated by an old stellar population (Jablonka, Martin 
\& Arimoto 1996), while the starbursts [SB] are dominated by young and massive stars, irrespective of their morphologies; c) From the stellar population viewpoint, the Seyfert galaxies do not form a homogeneous sample; d) We identify 3 regions related to 3 different dominant populations: [E] old population, [L] intermediate-age and young population, [SB] young and massive stars. In the HCGs, the LLAGNs and the galaxies without emission have smaller values of EW than normal galaxies for their morphologies, suggesting the presence of intermediate-age stellar population.

Fig. 6. - EW-EW diagram for the G-band as a function of the $\mathrm{CN}$ line. The same conclusion as in Figure 4d is reached for the HCG galaxies.

Fig. 7.- The EW $(\mathrm{H} \delta)$ as a function of the ratio of the intensities of lines CaII $\mathrm{H}+\mathrm{H} \epsilon$ over CaII K. Typical values for normal galaxies of different morphologies are indicated (Barbaro $\&$ Poggianti 1997). The two vertical lines indicate the ratios CaII H $+\mathrm{H} \epsilon$ for an old stellar population with $30 \%$ and no A stars present (Rose 1985). All the HCG galaxies have strong Balmer EW and relatively small CaII $\mathrm{H}+\mathrm{H} \epsilon / \mathrm{CaII} \mathrm{K}$ ratio, consistent with the evolved post-starbursts hypothesis.

Fig. 8. - The $\mathrm{Mg}_{2}$ index as a stellar metallicity indicator. a) The stellar population in normal galaxies trace a sequence of metallicities, which increase towards the early-type galaxies; b) The stellar metallicities of the HCG galaxies are compared to those of normal galaxies; the mean metallicities and the range values for normal early $(\mathrm{T} \leq 0)$ and late-type galaxies are represented by two boxes. The HCG galaxies without morphological information were placed at the two extremes of the figure. Typical uncertainty is indicated as error bar at the right of the figure.

Fig. 9.- The $\mathrm{Mg}_{2}$ index in function of the luminosities. The HCG galaxies are compared with the early-type galaxies from Bender, Burstein \& Faber (1989; BBF) and the bulge of 
spirals from Jablonka, Martin \& Arimoto (1996; JMA). The solid line is a linear fit on the Bender, Burstein \& Faber data. The dashed line is the same fit offset by +0.04 mag. We also show the error bar on our measures. The values of $\mathrm{Mg}_{2}$ of $6 \mathrm{HCG}$ galaxies as observed by Jorgensen (1997) are also shown for comparison.

Fig. 10.- Frequency distribution of the peculiar velocities of the emission-line galaxies relative to their group mean velocity. The AGNs and the galaxies without emission show a clear tendency to have smaller peculiar velocities than the starbursts. 
Table 1. Spectral line ratios relative to $\mathrm{H} \alpha$

\begin{tabular}{|c|c|c|c|c|c|c|c|c|c|c|c|}
\hline HCG \# & $\lambda 4101$ & $\lambda 4340$ & $\lambda 4363$ & $\lambda 4471$ & $\lambda 4861$ & $\lambda 5007$ & $\lambda 5876$ & $\lambda 6300$ & $\lambda 6584$ & $\lambda 6717$ & $\lambda 6731$ \\
\hline 0401 & 0.84 & 4.25 & $\ldots$ & $\cdots$ & 21.19 & 2.04 & 3.63 & 1.17 & 49.05 & 8.87 & 6.92 \\
\hline 0403 & $\ldots$ & $\ldots$ & $\ldots$ & $\ldots$ & 17.75 & 7.60 & $\ldots$ & 2.44 & 47.48 & 12.29 & 8.68 \\
\hline 0411 & 5.65 & 13.48 & 2.87 & 2.82 & 46.56 & 271.77 & 6.86 & 1.10 & 5.43 & 4.50 & 4.70 \\
\hline \multirow[t]{2}{*}{1601} & $\ldots$ & $\ldots$ & $\cdots$ & $\cdots$ & 7.8 & 16.0 & $\cdots$ & 13.5 & 123.5 & 29.9 & 22.3 \\
\hline & & & & & \pm 0.9 & 0.6 & & \pm 0.5 & \pm 0.3 & \pm 0.4 & \pm 0.5 \\
\hline \multirow[t]{2}{*}{1602} & $\ldots$ & 3 & $\ldots$ & $\ldots$ & 9.8 & 37.1 & $\ldots$ & 39.8 & 111.2 & 95.6 & 28.1 \\
\hline & & \pm 1 & & & \pm 0.7 & \pm 0.4 & & \pm 0.4 & \pm 0.3 & \pm 0.3 & \pm 0.4 \\
\hline \multirow[t]{2}{*}{1603} & $\ldots$ & $\ldots$ & $\ldots$ & $\ldots$ & 8.9 & 11.8 & 5.6 & 2.7 & 44.4 & 23.4 & 14.5 \\
\hline & & & & & \pm 0.3 & \pm 0.2 & \pm 0.3 & \pm 0.5 & \pm 0.1 & \pm 0.2 & \pm 0.2 \\
\hline 1604 & 1.14 & 4.15 & $\ldots$ & $\ldots$ & 18.60 & 8.33 & 4.30 & 1.2 & 41.48 & 16.42 & 10.60 \\
\hline \multirow[t]{2}{*}{1605} & $\ldots$ & $\ldots$ & $\ldots$ & $\cdots$ & 3.6 & 8.1 & 3.6 & 7.3 & 59.9 & 22.2 & 14.5 \\
\hline & & & & & \pm 0.4 & 0.2 & 0.4 & 0.3 & 0.1 & 0.1 & 0.2 \\
\hline \multirow[t]{2}{*}{1606} & $\ldots$ & $\ldots$ & $\ldots$ & $\cdots$ & 14 & 56 & $\ldots$ & 8 & 16 & 46 & 39 \\
\hline & & & & & \pm 2 & \pm 1 & & \pm 3 & \pm 2 & \pm 1 & \pm 1 \\
\hline \multirow[t]{2}{*}{2201} & $\ldots$ & $\ldots$ & $\ldots$ & $\ldots$ & 26 & 80 & $\ldots$ & 11 & 107 & 21 & 31 \\
\hline & & & & & \pm 3 & \pm 2 & & \pm 4 & \pm 2 & \pm 3 & \pm 2 \\
\hline 2303 & $\ldots$ & $\cdots$ & $\ldots$ & $\cdots$ & 26.52 & 52.61 & $\ldots$ & 32.26 & 163.00 & $\cdots$ & $\cdots$ \\
\hline 2304 & 2.107 & 5.536 & $\ldots$ & $\cdots$ & 22.20 & 12.39 & 4.74 & 1.66 & 40.74 & $\ldots$ & $\cdots$ \\
\hline \multirow[t]{2}{*}{2305} & $\ldots$ & $\ldots$ & $\ldots$ & $\ldots$ & \pm 8.34 & 24.58 & $\cdots$ & 1.0 & 81.70 & $\ldots$ & $\cdots$ \\
\hline & & & & & \pm 0.05 & \pm 0.03 & & \pm 0.1 & \pm 0.02 & & \\
\hline \multirow[t]{2}{*}{4001} & $\ldots$ & $\ldots$ & $\ldots$ & $\ldots$ & 18 & 18 & $\ldots$ & $\ldots$ & 87 & $\ldots$ & $\ldots$ \\
\hline & & & & & \pm 4 & \pm 4 & & & \pm 2 & & \\
\hline \multirow[t]{2}{*}{4004} & $\ldots$ & $\ldots$ & $\ldots$ & $\cdots$ & 9 & 12 & $\ldots$ & 14 & 65 & $\ldots$ & $\cdots$ \\
\hline & & & & & \pm 5 & \pm 4 & & \pm 4 & \pm 2 & & \\
\hline \multirow[t]{2}{*}{4005} & $\ldots$ & $\ldots$ & $\ldots$ & $\ldots$ & 11 & 30 & $\ldots$ & $\ldots$ & 60 & $\ldots$ & $\ldots$ \\
\hline & & & & & \pm 2 & \pm 2 & & & \pm 1 & & \\
\hline 4201 & $\ldots$ & $\ldots$ & $\ldots$ & $\cdots$ & $\ldots$ & $\ldots$ & $\cdots$ & $\ldots$ & 178 & $\ldots$ & $\cdots$ \\
\hline 6201 & $\ldots$ & $\ldots$ & $\ldots$ & $\ldots$ & 16 & 16 & $\ldots$ & 8 & 60.1 & 24 & 24 \\
\hline
\end{tabular}


Table 1-Continued

\begin{tabular}{|c|c|c|c|c|c|c|c|c|c|c|c|}
\hline HCG \# & $\lambda 4101$ & $\lambda 4340$ & $\lambda 4363$ & $\lambda 4471$ & $\lambda 4861$ & $\lambda 5007$ & $\lambda 5876$ & $\lambda 6300$ & $\lambda 6584$ & $\lambda 6717$ & $\lambda 6731$ \\
\hline & & & & & \pm 1 & \pm 1 & & \pm 2 & \pm 0.9 & \pm 1 & \pm 1 \\
\hline \multirow[t]{2}{*}{6702} & $\ldots$ & $\ldots$ & $\ldots$ & $\ldots$ & 17 & 12 & $\ldots$ & $\cdots$ & 34 & $\ldots$ & 9 \\
\hline & & & & & \pm 1 & \pm 2 & & & \pm 1 & \pm 1 & \pm 2 \\
\hline \multirow[t]{2}{*}{6706} & $\ldots$ & $\ldots$ & $\cdots$ & $\ldots$ & 33 & 23 & $\ldots$ & $\cdots$ & 37 & $\ldots$ & $\ldots$ \\
\hline & & & & & \pm 1 & \pm 1 & & & \pm 1 & & \\
\hline \multirow[t]{2}{*}{6711} & $\cdots$ & $\cdots$ & $\cdots$ & $\cdots$ & 182 & 211 & $\ldots$ & 99 & 515 & $\ldots$ & $\cdots$ \\
\hline & & & & & \pm 31 & \pm 32 & & \pm 29 & \pm 47 & & \\
\hline \multirow[t]{2}{*}{8601} & $\cdots$ & $\ldots$ & $\ldots$ & $\ldots$ & 56 & 23 & $\cdots$ & 23 & 62 & $\ldots$ & $\cdots$ \\
\hline & & & & & \pm 4 & \pm 5 & & \pm 5 & \pm 4 & & \\
\hline \multirow[t]{2}{*}{8603} & $\cdots$ & $\ldots$ & $\ldots$ & $\ldots$ & 16 & 145 & $\ldots$ & 8 & 82 & $\cdots$ & $\cdots$ \\
\hline & & & & & \pm 2 & \pm 1 & & \pm 3 & \pm 1 & & \\
\hline \multirow[t]{2}{*}{8604} & $\cdots$ & $\ldots$ & $\ldots$ & $\ldots$ & 15 & 23 & $\ldots$ & 15 & 71.0 & $\ldots$ & $\cdots$ \\
\hline & & & & & \pm 1 & \pm 1 & & \pm 1 & \pm 0.8 & & \\
\hline \multirow[t]{2}{*}{8607} & $\ldots$ & $\ldots$ & $\ldots$ & $\ldots$ & 17 & 248 & $\ldots$ & 43 & 123.9 & $\ldots$ & $\cdots$ \\
\hline & & & & & \pm 2 & \pm 1 & & \pm 1 & \pm 0.9 & & \\
\hline \multirow[t]{2}{*}{8608} & $\ldots$ & $\ldots$ & $\ldots$ & $\ldots$ & 10.2 & 4.6 & $\ldots$ & 3.2 & 33.6 & 22.9 & 14.6 \\
\hline & & & & & \pm 0.3 & \pm 0.5 & & \pm 0.6 & \pm 0.2 & \pm 0.2 & \pm 0.3 \\
\hline \multirow[t]{2}{*}{8609} & $\ldots$ & $\ldots$ & $\ldots$ & $\ldots$ & 13.2 & 10.5 & $\ldots$ & $\cdots$ & 22.0 & $\ldots$ & $\cdots$ \\
\hline & & & & & \pm 0.8 & \pm 0.9 & & & \pm 0.6 & & \\
\hline 8701 & $\ldots$ & $\ldots$ & $\ldots$ & $\ldots$ & 24.20 & 28.96 & $\ldots$ & 31.60 & 82.73 & $\ldots$ & $\cdots$ \\
\hline 8703 & $\ldots$ & $\ldots$ & $\ldots$ & $\ldots$ & 50.78 & 22.76 & $\ldots$ & $\ldots$ & 128.64 & $\ldots$ & $\ldots$ \\
\hline 8704 & $\cdots$ & $\ldots$ & $\cdots$ & $\ldots$ & 22.09 & 9.28 & $\cdots$ & $\ldots$ & 43.77 & $\cdots$ & $\cdots$ \\
\hline 8801 & $\cdots$ & $\ldots$ & $\cdots$ & $\cdots$ & 14.96 & 45.13 & $\ldots$ & 10.02 & 89.49 & $\ldots$ & $\cdots$ \\
\hline 8802 & $\cdots$ & $\ldots$ & $\cdots$ & $\ldots$ & 23.59 & 36.02 & $\ldots$ & 7.72 & 191.80 & $\cdots$ & $\cdots$ \\
\hline 8807 & $\cdots$ & $\cdots$ & $\cdots$ & $\ldots$ & 18.38 & 23.28 & $\ldots$ & $\cdots$ & 32.60 & $\ldots$ & $\cdots$ \\
\hline \multirow[t]{2}{*}{9001} & $\cdots$ & $\ldots$ & $\ldots$ & $\ldots$ & 16 & 144 & $\ldots$ & $\ldots$ & 120 & 36 & 38 \\
\hline & & & & & \pm 3 & \pm 2 & & & \pm 2 & \pm 2 & \pm 2 \\
\hline 9004 & $\ldots$ & $\ldots$ & $\ldots$ & $\ldots$ & 8 & 8 & $\ldots$ & 8 & 62.7 & 18 & 12 \\
\hline
\end{tabular}


Table 1-Continued

\begin{tabular}{|c|c|c|c|c|c|c|c|c|c|c|c|}
\hline $\mathrm{HCG} \#$ & $\lambda 4101$ & $\lambda 4340$ & $\lambda 4363$ & $\lambda 4471$ & $\lambda 4861$ & $\lambda 5007$ & $\lambda 5876$ & $\lambda 6300$ & $\lambda 6584$ & $\lambda 6717$ & $\lambda 6731$ \\
\hline & & & & & \pm 2 & \pm 1 & & \pm 2 & \pm 0.6 & \pm 1 & \pm 1 \\
\hline \multirow[t]{2}{*}{9009} & $\ldots$ & $\ldots$ & $\ldots$ & $\ldots$ & 27.4 & 44.1 & $\ldots$ & $\ldots$ & 24.8 & $\ldots$ & 14.2 \\
\hline & & & & & \pm 0.7 & \pm 0.6 & & & \pm 0.7 & \pm 0.6 & \pm 0.9 \\
\hline \multirow[t]{2}{*}{9705} & $\ldots$ & $\cdots$ & $\ldots$ & $\ldots$ & 23 & 40 & $\cdots$ & $\ldots$ & 53 & $\ldots$ & $\ldots$ \\
\hline & & & & & \pm 3 & \pm 2 & & & \pm 2 & & \\
\hline \multirow[t]{2}{*}{9706} & $\cdots$ & $\ldots$ & $\ldots$ & $\cdots$ & 16 & 39 & $\cdots$ & $\cdots$ & 56 & $\cdots$ & $\cdots$ \\
\hline & & & & & \pm 2 & \pm 2 & & & \pm 2 & & \\
\hline
\end{tabular}


Table 2. Characteristics of HCG emission-line galaxies

\begin{tabular}{|c|c|c|c|c|c|c|}
\hline HCG \# & B & $\begin{array}{c}\mathrm{cz} \\
\left(\mathrm{km} \mathrm{s}^{-1}\right)\end{array}$ & $\mathrm{M}_{\mathrm{B}}$ & $\mathrm{T}$ & $\begin{array}{c}\text { Activity } \\
\text { Type }\end{array}$ & $\begin{array}{c}\mathrm{EW} \\
\mathrm{H} \alpha+[\mathrm{NII}] \\
(\AA)\end{array}$ \\
\hline 0401 & 13.71 & 8035 & -21.44 & 5 & $\mathrm{SBNG}$ & 106 \\
\hline 0403 & 15.97 & 8242 & -19.23 & -5 & SBNG & 44 \\
\hline 0411 & 17.75 & 6957 & -17.09 & $\ldots$ & HII & 261 \\
\hline 1601 & 12.88 & 4073 & -20.79 & 2 & LNR & 10 \\
\hline 1602 & 13.35 & 3864 & -20.21 & 2 & Sy2 & 18 \\
\hline 1603 & 13.35 & 4001 & -20.29 & $\cdots$ & SBNG & 69 \\
\hline 1604 & 13.61 & 3859 & -19.95 & 10 & SBNG & 154 \\
\hline 1605 & 13.66 & 3934 & -19.94 & $\ldots$ & LNR & 74 \\
\hline 1606 & 15.56 & 3972 & -18.06 & $\cdots$ & HII & 14 \\
\hline 2201 & 12.60 & 2681 & -20.17 & -5 & dSy 2 & 2 \\
\hline 2302 & 15.03 & 9899 & -20.57 & $\cdots$ & Sy2 & 28 \\
\hline 2303 & 15.21 & 4869 & -18.85 & 2 & $\mathrm{dLNR}$ & 5 \\
\hline 2304 & 15.81 & 4467 & -18.06 & 7 & SBNG & 181 \\
\hline 2305 & 16.14 & 5373 & -18.14 & -2 & $\mathrm{dSy} 2$ & 5 \\
\hline 4001 & 14.32 & 6634 & -20.41 & -5 & dLNR & 4 \\
\hline 4004 & 15.20 & 6362 & -19.44 & 1 & LNR & 13 \\
\hline 4005 & 16.50 & 6633 & -18.23 & 5 & Sy2 & 25 \\
\hline 4201 & 12.62 & 3712 & -20.85 & -5 & dLNR & 4 \\
\hline 6201 & 13.30 & 4259 & -20.47 & -5 & dLNR & 5 \\
\hline 6702 & 14.57 & 7543 & -20.44 & 5 & SBNG & 28 \\
\hline 6706 & 15.66 & 7831 & -19.43 & $\ldots$ & SBNG & 21 \\
\hline 6711 & 16.49 & 6614 & -18.24 & $\cdots$ & dLNR & 3 \\
\hline 8601 & 14.23 & 6013 & -20.29 & -5 & dLNR & 2 \\
\hline 8603 & 14.81 & 5863 & -19.66 & -5 & $\mathrm{dSy} 2$ & 5 \\
\hline 8604 & 15.30 & 5298 & -18.95 & -2 & LNR & 8 \\
\hline 8607 & 15.98 & 6476 & -18.70 & $\ldots$ & Sy2 & 13 \\
\hline 8608 & 16.20 & 5508 & -18.13 & $\cdots$ & SBNG & 90 \\
\hline 8609 & 16.26 & 6816 & -18.53 & $\ldots$ & SBNG & 19 \\
\hline
\end{tabular}


Table 2-Continued

\begin{tabular}{lcccccc}
\hline \hline HCG \# & B & cz & M & T & Activity & EW \\
& & $\left(\mathrm{km} \mathrm{s}^{-1}\right)$ & & & Type & H $\alpha+[\mathrm{NII}]$ \\
& & & & & & $(\AA)$ \\
\hline 8701 & 14.81 & 8436 & -20.45 & 4 & dLNR & 6 \\
8703 & 15.40 & 8738 & -19.93 & 0 & dLNR & 2 \\
8704 & 15.78 & 8963 & -19.61 & 4 & SBNG & 19 \\
8801 & 13.71 & 6007 & -20.81 & 3 & dSy2 & 7 \\
8802 & 13.81 & 6124 & -20.75 & 3 & dLNR & 10 \\
9001 & 12.99 & 2603 & -19.71 & 1 & Sy2 & 8 \\
9004 & 13.54 & 2659 & -19.21 & $\ldots$ & LNR & 15 \\
9009 & 16.80 & 2760 & -16.03 & $\ldots$ & SBNG & 43 \\
9705 & 15.29 & 6379 & -19.36 & $\ldots$ & LNR & 11 \\
9706 & 15.45 & 6666 & -19.29 & 6 & Sy2 & 17 \\
\hline & & & & & & \\
\hline
\end{tabular}


Table 3. Absorption features in HCG AGNs

\begin{tabular}{ccccccccccc}
\hline \hline HCG \# & $\begin{array}{c}\text { CaII K } \\
(\AA)\end{array}$ & $\begin{array}{c}\text { CaII H } \\
(\AA)\end{array}$ & $\begin{array}{c}\mathrm{H} \delta \\
(\AA)\end{array}$ & $\begin{array}{c}\mathrm{CN} \\
(\AA)\end{array}$ & $\begin{array}{c}\text { G-band } \\
(\AA)\end{array}$ & $\begin{array}{c}\mathrm{H} \beta \\
(\AA)\end{array}$ & $\begin{array}{c}\mathrm{Mg} \\
(\AA)\end{array}$ & $\begin{array}{c}\mathrm{Na} \\
(\AA)\end{array}$ & $\frac{\mathrm{I}(\mathrm{C} \text { (aIIH })}{\mathrm{I}(\mathrm{CaIIK})}$ & $\mathrm{Mg}_{2}$ \\
& $\ldots$ & $\ldots$ & & & & & & & & \\
1601 & $\ldots$ & $\ldots$ & 1.9 & 4.9 & 4.1 & 3.7 & 5.7 & $\ldots$ & 0.21 \\
1602 & $\ldots$ & $\ldots$ & 1.4 & 2.9 & 8.4 & $\ldots$ & 4.9 & 7.6 & $\ldots$ & 0.33 \\
1605 & $\ldots$ & $\ldots$ & 6.0 & 2.9 & 2.7 & 5.7 & 1.7 & 4.4 & $\ldots$ & $\ldots$ \\
4004 & 6.28 & 2.62 & $\ldots$ & 4.1 & 5.1 & $\ldots$ & 4.6 & 4.8 & 1.0 & 0.19 \\
4005 & $\ldots$ & $\ldots$ & 2.6 & 4.7 & 6.6 & $\ldots$ & 4.6 & $\ldots$ & $\ldots$ & 0.22 \\
8604 & $\ldots$ & $\ldots$ & 2.7 & 2.4 & 8.9 & 2.6 & 5.5 & 5.2 & $\ldots$ & 0.33 \\
8607 & 9.08 & 5.78 & 2.4 & 2.0 & 4.3 & 4.0 & 3.5 & 3.6 & 1.1 & 0.13 \\
9001 & $\ldots$ & $\ldots$ & 2.3 & 4.1 & 7.1 & 4.0 & 4.3 & 3.7 & $\ldots$ & 0.17 \\
9004 & $\ldots$ & $\ldots$ & 1.7 & 4.8 & 5.5 & 2.9 & 4.4 & 4.2 & $\ldots$ & 0.19 \\
\hline
\end{tabular}


Table 4. Absorption features in HCG LLAGNs

\begin{tabular}{lcccccccccc}
\hline \hline HCG \# & CaII K & CaII H & $\mathrm{H} \delta$ & $\mathrm{CN}$ & $\begin{array}{c}\text { G-band } \\
(\AA)\end{array}$ & $\begin{array}{c}\mathrm{H} \beta \\
(\AA)\end{array}$ & $\begin{array}{c}\mathrm{Mg} \\
(\AA)\end{array}$ & $\begin{array}{c}\mathrm{Na} \\
(\AA)\end{array}$ & $\begin{array}{c}\mathrm{I}(\mathrm{CaIIH}) \\
\mathrm{I}(\mathrm{CaIIK})\end{array}$ & $\mathrm{Mg}_{2}$ \\
\hline & & & & & & $\AA$ & $(\AA)$ & $(\AA)$ & & \\
2201 & $\ldots$ & $\ldots$ & 2.2 & 6.0 & 7.0 & 2.9 & 4.9 & 5.4 & $\ldots$ & 0.33 \\
2303 & $\ldots$ & $\ldots$ & 2.2 & 4.1 & 6.4 & 2.2 & 4.3 & 4.6 & $\ldots$ & 0.29 \\
2305 & $\ldots$ & $\ldots$ & 3.8 & 2.4 & 2.3 & 5.6 & 3.0 & 4.2 & $\ldots$ & 0.13 \\
4001 & 12.0 & 8.6 & 2.9 & 1.8 & 6.6 & 3.6 & 4.3 & 6.1 & 1.1 & 0.26 \\
4201 & $\ldots$ & $\ldots$ & 1.8 & 2.5 & 7.5 & 2.1 & 4.8 & 9.3 & $\ldots$ & 0.34 \\
6201 & $\ldots$ & $\ldots$ & 1.8 & 2.8 & 6.2 & 1.7 & 4.8 & 6.9 & $\ldots$ & 0.33 \\
6711 & 9.3 & 7.8 & 2.4 & 2.5 & 6.6 & 2.0 & 4.8 & 5.2 & 1.0 & 0.34 \\
8601 & 11.2 & 9.2 & 2.0 & 2.5 & 6.1 & 2.5 & 4.9 & 8.9 & 1.1 & 0.35 \\
8603 & 9.9 & 6.6 & 1.6 & 2.6 & 7.0 & 2.8 & 5.1 & 5.8 & 1.0 & 0.31 \\
8701 & 5.7 & 6.3 & 1.5 & 3.8 & 4.9 & 1.3 & 3.8 & 6.1 & 1.0 & 0.25 \\
8703 & 12.2 & 8.2 & 2.1 & 2.9 & 7.2 & 1.9 & 5.4 & 5.8 & 1.1 & 0.31 \\
8801 & 13.0 & 9.0 & 1.9 & 4.4 & 5.6 & 5.1 & 3.9 & 5.4 & 1.1 & 0.25 \\
8802 & 12.3 & 11.1 & 2.0 & 2.4 & 7.5 & 3.0 & 4.4 & 4.0 & 1.1 & 0.23 \\
\hline
\end{tabular}


Table 5. Absorption features in HCG starbursts

\begin{tabular}{|c|c|c|c|c|c|c|c|c|c|c|}
\hline HCG \# & $\begin{array}{c}\text { CaII K } \\
(\AA)\end{array}$ & $\begin{array}{c}\text { CaII H } \\
(\AA)\end{array}$ & $\begin{array}{l}\mathrm{H} \delta \\
(\AA)\end{array}$ & $\begin{array}{l}\mathrm{CN} \\
(\AA)\end{array}$ & $\begin{array}{c}\text { G-band } \\
(\AA)\end{array}$ & $\begin{array}{l}\mathrm{H} \beta \\
(\AA)\end{array}$ & $\begin{array}{l}\mathrm{Mg} \\
(\AA)\end{array}$ & $\begin{array}{l}\mathrm{Na} \\
(\AA)\end{array}$ & $\frac{\mathrm{I}(\mathrm{CaIIH})}{\mathrm{I}(\mathrm{CaIIK})}$ & $\mathrm{Mg}_{2}$ \\
\hline 0401 & 2.5 & 2.2 & em & 1.6 & 2.9 & em & 1.6 & 1.4 & 1.0 & $\ldots$ \\
\hline 0403 & 3.8 & 5.9 & 4.4 & 2.0 & 2.7 & 4.1 & 2.8 & 1.4 & 1.0 & 0.09 \\
\hline 0411 & $\ldots$ & $\ldots$ & em & 3.7 & $\ldots$ & em & $\ldots$ & $\ldots$ & $\ldots$ & 0.27 \\
\hline 1603 & $\ldots$ & $\ldots$ & 5.8 & 2.5 & 3.0 & 5.2 & 2.4 & 2.4 & $\ldots$ & $\ldots$ \\
\hline 1604 & $\ldots$ & $\ldots$ & 3.3 & 1.0 & 0.6 & em & 1.4 & 2.9 & $\ldots$ & $\ldots$ \\
\hline 1606 & $\ldots$ & $\ldots$ & 3.2 & 3.5 & 4.7 & 3.1 & $\ldots$ & $\ldots$ & $\ldots$ & $\ldots$ \\
\hline 2304 & $\ldots$ & $\ldots$ & em & 1.8 & 1.5 & em & 1.7 & 0.8 & $\ldots$ & $\ldots$ \\
\hline 6306 & $\ldots$ & $\ldots$ & em & 2.3 & 4.3 & em & 2.6 & 4.4 & $\ldots$ & 0.09 \\
\hline 6702 & $\ldots$ & $\ldots$ & $\ldots$ & 4.7 & 5.6 & em & 4.9 & 2.3 & $\ldots$ & 0.21 \\
\hline 8605 & 10.7 & 5.8 & 2.8 & $\cdots$ & 5.2 & 3.7 & 3.6 & 4.5 & 0.9 & 0.21 \\
\hline 8608 & $\ldots$ & $\ldots$ & 4.2 & 1.5 & $\ldots$ & em & $\ldots$ & 3.8 & $\ldots$ & $\ldots$ \\
\hline 8609 & $\ldots$ & $\ldots$ & 1.9 & 5.7 & 5.1 & 1.6 & 4.7 & 3.9 & $\ldots$ & 0.19 \\
\hline 8702 & $\ldots$ & $\ldots$ & $\ldots$ & 4.0 & $\ldots$ & em & 2.5 & $\ldots$ & $\ldots$ & $\ldots$ \\
\hline 8705 & $\ldots$ & $\ldots$ & $\ldots$ & 2.2 & $\ldots$ & em & $\ldots$ & $\ldots$ & $\ldots$ & $\ldots$ \\
\hline 8707 & 3.8 & 4.2 & 4.4 & 2.0 & 2.2 & 4.3 & 2.1 & 2.3 & 1.0 & $\ldots$ \\
\hline 8807 & 6.2 & 7.8 & em & 4.8 & 7.0 & 4.4 & 3.5 & 3.2 & 1.3 & 0.34 \\
\hline
\end{tabular}


Table 6. Absorption features in HCG galaxies without emission

\begin{tabular}{cccccccccccc}
\hline \hline HCG \# & CaII K & CaII H & $\mathrm{H} \delta$ & $\mathrm{CN}$ & $\mathrm{G}-$ band & $\mathrm{H} \beta$ & $\mathrm{Mg}$ & $\mathrm{Na}$ & $\frac{\mathrm{I}(\mathrm{CaIIH})}{\mathrm{I}(\mathrm{CaIIK})}$ & $\mathrm{Mg}_{2}$ & $\mathrm{~T}$ \\
& $(\AA)$ & $(\AA)$ & $(\AA)$ & $(\AA)$ & $(\AA)$ & $(\AA)$ & $(\AA)$ & $(\AA)$ & & & \\
\hline & & & & & & & & & & & \\
1901 & $\ldots$ & $\ldots$ & 2.3 & 2.5 & 6.5 & 3.1 & 4.5 & 3.6 & $\ldots$ & 0.27 & -5 \\
2203 & $\ldots$ & $\ldots$ & 3.7 & 2.7 & 4.1 & 4.9 & 3.6 & 1.9 & $\ldots$ & 0.16 & $\ldots$ \\
2204 & $\ldots$ & $\ldots$ & 3.8 & 2.1 & 6.6 & 4.1 & 3.6 & 2.9 & $\ldots$ & 0.17 & 1 \\
4002 & 12.6 & 7.7 & 2.7 & 2.7 & 5.9 & 3.5 & 4.1 & 6.0 & 1.0 & 0.26 & -2 \\
4202 & $\ldots$ & $\ldots$ & $\ldots$ & 3.5 & 6.9 & 2.4 & 4.7 & 5.4 & $\ldots$ & 0.30 & -2 \\
4204 & $\ldots$ & $\ldots$ & 2.8 & 3.6 & 6.4 & 5.1 & 4.0 & 5.7 & $\ldots$ & 0.29 & -5 \\
4801 & $\ldots$ & $\ldots$ & 2.7 & 3.4 & 8.5 & 3.0 & 4.3 & 5.0 & $\ldots$ & 0.32 & -5 \\
6202 & $\ldots$ & $\ldots$ & 2.4 & 1.9 & 6.2 & 3.3 & 4.9 & 6.5 & $\ldots$ & 0.32 & -2 \\
6203 & $\ldots$ & $\ldots$ & 1.4 & 2.7 & 6.0 & 1.9 & 4.9 & 4.1 & $\ldots$ & 0.27 & -2 \\
6204 & $\ldots$ & $\ldots$ & 2.2 & 3.3 & 7.2 & 2.5 & 4.5 & 3.9 & $\ldots$ & 0.29 & \\
6208 & $\ldots$ & $\ldots$ & 2.8 & 5.2 & 8.0 & 2.8 & 4.7 & 3.5 & $\ldots$ & 0.26 & $\ldots$ \\
6309 & 4.4 & 3.6 & 2.0 & 2.5 & 5.5 & 1.7 & 4.9 & 5.3 & 1.1 & 0.27 & $\ldots$ \\
6701 & 10.5 & 6.2 & 1.4 & 3.0 & 7.1 & 1.7 & 4.4 & 5.5 & 1.1 & 0.30 & -5 \\
6703 & 10.6 & 6.9 & 2.5 & 3.5 & 7.0 & 2.5 & 5.1 & 4.3 & 1.0 & 0.28 & $\ldots$ \\
8602 & 12.1 & 9.1 & 2.6 & 1.4 & 7.2 & 3.5 & 4.8 & 7.5 & 1.1 & 0.33 & $\ldots$ \\
8606 & 11.9 & 8.2 & 1.7 & 2.9 & 6.7 & 2.4 & 4.0 & 4.9 & 1.0 & 0.26 & -2 \\
9003 & $\ldots$ & $\ldots$ & 1.7 & 2.6 & 8.3 & 1.5 & 5.0 & 4.9 & $\ldots$ & 0.29 & -5 \\
9702 & 10.8 & 8.2 & 2.8 & 2.3 & 5.8 & 4.9 & 3.7 & 5.3 & 1.1 & 0.24 & $\ldots$ \\
9710 & 14.2 & 9.8 & 2.9 & 3.3 & 7.5 & 3.1 & 4.6 & 5.2 & 1.0 & 0.31 & $\ldots$ \\
9711 & 6.6 & 6.5 & 2.8 & 3.4 & 6.6 & 3.0 & 3.7 & 4.0 & 1.1 & 0.25 & $\ldots$ \\
\hline & & & & & & & & & & &
\end{tabular}


Table 7. Electron densities and metallicities of HCG Starbursts

\begin{tabular}{|c|c|c|c|}
\hline HCG \# & $\frac{\lambda 6717}{\lambda 6731}$ & $\begin{array}{c}\mathrm{N}_{e} \\
\left(\mathrm{~cm}^{-3}\right)\end{array}$ & $12+\log (\mathrm{O} / \mathrm{H})$ \\
\hline 0401 & 1.5 & 110 & 8.9 \\
\hline 0403 & 1.4 & 70 & 9.0 \\
\hline 0411 & 1.0 & 800 & 8.1 \\
\hline 1603 & 1.6 & $<10$ & 8.6 \\
\hline 1604 & 1.6 & $<10$ & 9.0 \\
\hline 1606 & 1.2 & 300 & 8.3 \\
\hline 1905 & 1.3 & 100 & 8.8 \\
\hline 2207 & $\ldots$ & $\ldots$ & 9.1 \\
\hline 2304 & $\ldots$ & & 8.9 \\
\hline 2306 & 1.5 & $<10$ & 8.8 \\
\hline 4819 & 1.4 & 70 & 8.9 \\
\hline 4825 & 1.6 & $<10$ & 8.5 \\
\hline 6306 & $\ldots$ & $\ldots$ & 9.0 \\
\hline 6422 & $\ldots$ & $\ldots$ & 8.8 \\
\hline 6702 & $\ldots$ & $\ldots$ & 8.8 \\
\hline 6706 & $\ldots$ & $\ldots$ & 8.8 \\
\hline 8608 & 1.6 & $<10$ & 9.0 \\
\hline 8609 & $\ldots$ & $\ldots$ & 8.7 \\
\hline 8702 & 1.1 & 600 & 9.0 \\
\hline 8705 & 1.2 & 300 & 8.5 \\
\hline 8707 & $\ldots$ & $\ldots$ & 8.7 \\
\hline 8807 & $\cdots$ & $\cdots$ & 8.6 \\
\hline 9009 & $\ldots$ & $\ldots$ & 8.6 \\
\hline
\end{tabular}

